\title{
Editorial \\ Weaving Trauma into the Everyday - \\ Neuroscience, Evidence and Reflections from Practice
}

Joe Tucci and Janise Mitchell

Last year, the Australian Childhood Foundation celebrated an important milestone. We turned thirty years old. For more than half of this period, we have sought to translate the richness of ideas that the field of neuroscience has offered to those of us taking care of children, young people and adults who have suffered trauma resulting from violation.

We have held the goal to collaborate with others to find compassion for the pain that children and young people carry with them as a result of the abuse and violence they have been forced to endure. Our whole field has been on an expedition into new territories of understanding, seeking to interpret the consequences of constant threat being embedded deeply into the brains and bodies of children and young people.

We have worked to apply the resources offered by Siegel, Schore, Tronick, Porges, Ogden, Atkinson, Van Der Kolk, Hughes, Teicher and others ways that are relevant and helpful for practitioners in education, child protection, out of home care, family support, youth justice and mental health.

Our first International Childhood Trauma Conference brought many of these speakers to Australia into one evocative and inspiring conversation in Melbourne in 2014. Our second Conference in June 2016 replicated the intensity and vibrancy of these reflections. More than 2500 people attended and drew from the passion and collective insights of each other and our international guests. This time, the discussions focussed their attention on the everyday realities of what it means to integrate the evidence from the neurobiology of attachment disruption and trauma into therapeutic approaches and practices.

The papers in this Special Edition of Children Australia are drawn from presentations given at the 2016 International Childhood Trauma Conference. They weave together themes that navigate the field of trauma intervention and neuroscience across a diverse set of parameters and areas of interest.
Cathy Crouch exquisitely describes the work she and her team at Mallee District Aboriginal Service have undertaken to develop a culturally sensitive resource supporting mothers to explore the sensory and lived experiences of their infants before and after they are born. Wondering from the $W o m b$ shares the wisdom of Aboriginal elders about antenatal health and safety so as to invite reflection from young parents on their way to the birth of their child.

Sideris carefully and painstakingly integrates trauma, cultural analysis and art therapy into a narrative about her work with a young boy deeply affected by violence. Her reflections provide an insight into the nature of transformation that occurs at the boundaries of connection and meaning making.

Jo Mensinga adopts a narrative methodology to explore the ways in which vicarious trauma shapes physiological experiences of those working in a family violence service. She finds and brings into scope stories that workers tell about their bodies as a means of understanding how to find resources that may keep them well and safe.

Arianne Struik explains her three phase model of intervention that works to transform the past of children and young people who have experienced complex trauma. She is committed to ensuring that challenging behaviour often displayed by children affected by trauma does not prevent them being offered effective forms of therapeutic support. Taking a related approach, Balu examines complex ecologies of trauma affected systems to offer ways of re-conceptualising interagency collaboration.

Bollinger, Scott Smith and Mendes explore the ways in which the youth justice system comes into contact with young people who have experienced complex trauma and come to live in residential out of home care services. This is a topical contribution to a current issue that faces the child protection system and broader community.

Costa has translated knowledge about trauma into educational settings, highlighting the importance of shifting 
school cultures in order to achieve sustained change for children and young people whose lives have been affected by toxic levels of stress and violence.

Finally, this issue is contains three book reviews. In addition to her article, Jenna Bollinger has offered her thoughts on the recently published book titled Young people transitioning from out-of-home care: International research, policy and practice, which is edited by Philip Mendes and Pamela Snow (2016). Kathy Mendis has provided a review of Sonia Jackson and Claire Cameron's (2014) book entitled Improving access to further and higher education for young people in public care: European policy and practice. And Di O'Neil has reviewed a book edited by May Crock (2015) called Creating new futures: Settling children and youth from refugee backgrounds.

These papers and the others which will follow in a second Special Edition in September highlight the need for a continued emphasis on the significance of the resources that neuroscience and the modalities, which it informs for supporting vulnerable children, young people and their families and carers.

However, there is some reason for concern. As we look across our field, it is as if we stand at a crossroad where the vigour and hope offered by neurobiology has started to wear off. And there are forces pushing us to find that next new "thing" - the next new form of intervention that will create change predictably and quickly. The pressure is palpable driven in part by influences such as government reform agendas, the preference of funding bodies, an impetus for efficiency and the need to respond to community concern about the way we have mistreated children in the past.

It is as if we have an in-built impatience with the significance of the power of attuned, sensitive and secure relationships in healing the hurt that traumatised children carry with them. It is likely the only experience that has ever done so.

If we are not careful, such impatience may push us towards the pragmatism of behavioural models that we know have not served traumatised children well in the past. It also may pull us towards a fragmentation of approaches, the emphasising of pathology and the failure to appreciate the strengths inherent in children's culture and the collective power of their community. Fragmentation itself is a product of trauma.

For this reason, it is our capacity to acknowledge that the insights from the research about trauma have changed our practice that will help us to attend to the past and ongoing unmet needs of children and young people who have been forced to live with violence. And in the face of their overwhelming distress, it may be the realisation that in turn we too have been changed that may give us the courage to do so.

The Third International Childhood Trauma Conference is set to take place in July 2018 in Melbourne. For more information about the speakers who are attending and how you can participate, visit www.childtraumaconf.org.

Dr Joe Tucci is CEO of the Australian Childhood Foundation. Janise Mitchell is the Deputy CEO of the Australian Childhood Foundation. 